\section{Mehr als normal - verstehen wir die Enhancement-Debatte?}

\author{
von Steffen Rosahl, Helios-Klinikum Erfurt
}

Der Nachmensch - ein mit Implantaten verstärkter Homo sapiens, dessen körperliche und geistige Fähigkeiten alles bisher menschlich Erreichbare übersteigen - erregt die Fantasie der Öffentlichkeit. Von den Einen gefürchtet, von den Anderen herbeigesehnt scheint er derzeit mehr Fiktion als wissenschaftliche Realität. Wenn die Entwicklung auf dem Gebiet neuroelektronischer Implantate linear verläuft, dann ist ein „Enhancement" mit Implantaten derzeit noch nicht abzusehen. Doch Linearität ist nicht immer die Regel und die sozialen und ökonomischen Dimensionen einer solchen Entwicklung wären derart massiv, dass die ethische Diskussion darüber schon heute weder zu unterlassen noch aufzuschieben ist.

Daniel starrte ungläubig auf den Aushang mit den Ergebnissen der schriftlichen Prüfung. Bisher war alles immer bestens für ihn gelaufen: Klassenbester, Teilnahme am Mathecamp, Zweiter der Geographie-Olympiade, Schulsieger in der English Challenge - selbst im Sport war es sein(!) Jahr gewesen. Eine Woge von Selbstvertrauen hatte ihn bis heute getragen und mit dem erwartet hervorragenden $A b$ schneiden bei den Prüfungen würde er sich locker einen Studienplatz an einer der besten Unis sichern - die Basis für einen Spitzenplatz in der Führungsriege der Gesellschaft.

Und jetzt das: In allen drei schriftlichen Prüfungen nur Mittelmaß - kein Fach besser als „, befriedigend" - Enttäuschung pur. Aber dann fiel sein Blick auf die Noten von Alex. Das war doch nicht möglich: 1,0 - eine glatte Eins in allen drei Prüfungen!

Daniel war immer gut mit seinem Zwillingsbruder ausgekommen. In der Familie hatte es klare Positionen gegeben: Alex war eher der Kumpeltyp - immer locker, jede Menge Freunde, Partys, ein paar Experimente mit Drogen, in der Schule nicht immer ganz aufmerksam, aber mit dem erreichten Mittelmaß ganz zufrieden. Er selbst war ehrgeiziger, in vielem begabter, auch fleißiger - in jedem Fall deutlich erfolgreicher als Alex. Sein Bruder schien ihm das nie zu neiden oder hatte er das nur versteckt? Vor einigen Monaten hatten sie einmal eine Diskussion...

„Was würdest Du sagen, wenn Dir jemand ein Implantat anbietet, mit dem Du Deine Gedächtnisleistung verzehnfachen kannst?", hatte Alex ihn gefragt.

,Was für ein Implantat meinst Du? Von Medikamenten hab ich schon gehört, die die Aufmerksamkeit und Reaktionsfähigkeit steigern, aber ein Implantat? Etwa im Gehirn? Wer erzählt denn so was? ", hatte er zurück gefragt.

„Ein Memory-Chip. Beste Voraussetzungen für das Abi. Garantierte Topleistung sonst Geld zurück!"

„Hört sich ja toll an - bloß schade, dass es so etwas nicht gibt. Schon gar nicht ohne Nebenwirkungen, soviel ist sicher."

„Nur ein paar kleine Nadeln im Gehirn, jederzeit abschaltbar. Würdest Du es wollen?", hatte Alex insistiert.

„Du spinnst mal wieder, Brüderchen, steck Deine Nase lieber noch mal in die Bücher - sonst wird die Matheklausur morgen Dein Nadelkissen!"

Damit war die Diskussion beendet und keiner von beiden war auf das Thema seitdem zurückgekommen. Alex hatte sich in den letzten Wochen rar gemacht und war oft mit seiner Clique losgezogen. Daniel hatte schon gefürchtet, dass sein Bruder durch die Prüfungen fliegen würde. Und jetzt waren unter den 50 Besten diesmal fast ausnahmslos Namen, die man dort noch nie gesehen hatte...

Sicher - das ist ein Science-Fiction-Szenario, aber wie weit hergeholt ist es wirklich? Jens Clausen hat in seinem Beitrag zu diesem Heft und schon zuvor gezeigt, dass es eine sichere ethische Grundlage für die Diskussion der Technologiefolgen medizinischer Implantate gibt (Clausen 2009). Die lange vor dem Belmont-Report (The National Commission for the Protection of Human Subjects of Biomedical and Behavioral Research 1979) formulierten ethisch-moralischen Prinzipien sind hier hilfreich und bedürfen allenfalls kleinerer Ergänzungen. Enhancement - die ,Verbesserung“ des Menschen durch Neurotechnologien - ist dabei zu Recht kein wirkliches Thema.

Vor zehn Jahren hat man sich entspannt zurücklehnen und die Diskussion wegen real 
fehlender Technologien verschieben können (White 1999) und ich selbst befinde mich in exzellenter Gesellschaft mit einer festen Überzeugung, dass man Dichtung und Wissenschaft gerade in diesem Zusammenhang sehr strikt auseinander halten sollte (Merkel et al. 2007; Rosahl 2007; Rosahl 2008). Zwei Fragen kann man dennoch nicht umgehen und verdrängen, wenn man ehrlich und wissenschaftlich glaubwürdig bleiben möchte:

1. Wie wahrscheinlich bzw. wie zeitlich nah ist eine technologische Schnittstelle, welche kognitive Fähigkeiten des Menschen über das bisher bekannte Maß bei optimaler Körperfunktion und in einem optimalem Milieu hinaus verstärken kann?

2. Haben wir bereits heute die ethischen Handwerkzeuge, um uns mit einer solchen Situation auseinander zu setzen?

Jede Wahrheit hat ihre Zeit und die öffentliche Debatte und Meinungsformung schert sich wenig darum, was Wissenschaftler glauben und wünschen. Fakten hingegen spielen eine gewisse Rolle und diese wiederum sind in Wissenschaft und Technologie ständiger Veränderung unterworfen. Die Neurochirurgie befindet sich dabei, vor allem wegen ihrer sehr direkten Beschäftigung mit dem biologischen Substrat menschlichen Denkens, in einer besonderen Position und zumindest ihren prominenteren Vertretern ist das sehr wohl bewusst (Elder et al. 2008).

Hat das Zeitalter der Cyborgs wirklich begonnen? Die mit neuroelektronischer Technologie verbundenen Ängste sind weitreichend. Sind sie auch gerechtfertigt? Betrachten wir die generellen technischen Voraussetzungen: Implantationen von langzeitig funktionellen Mikroelektroden in der Hirnrinde erscheinen bereits im Rahmen des Möglichen (Suner et al. 2005). Durch Beschichten und Bewachsen der Oberflächen werden sich hier in naher Zukunft noch deutliche Verbesserungen ergeben. Kochleäre und andere sensorische Implantate haben zumindest gezeigt, dass technische Schnittstellen zum Nervengewebe kein schwerwiegendes technologisches Problem mehr darstellen. ${ }^{1}$ Solange diese Implantate allerdings am Rezeptor (Cochlea, Retina etc.) enden und die gleichen Reizqualitäten aus der Umwelt (hörbarer Schall, sichtbares Licht) umformen und an das Gehirn weiterleiten sollen wie die natürlichen Sinnesorgane, besteht der gesamte Transmissionsprozess mehr oder weniger in einer Balancierung von SignalRausch-Verhältnissen. Wir haben Ertaubten das Hören zurückgegeben, aber wie geht es weiter? Sehprothesen mit großflächigen Mikroelektrodenarrays zur Stimulation der Retina oder des visuellen Kortex für zwei- oder dreidimensionale Erkennungsmuster werden im sensorischen Bereich die Grenzen für Schnittstellen zwischen neuronalem Gewebe und Elektronik erweitern. Durch mathematische Modellierung kognitiver Prozesse werden biohybride Computer möglich (Katz 2008; Sinclair et al. 2006). Biohybride elektronische Bauteile, die auf denselben Verarbeitungsstrategien aufbauen wie das menschliche Gehirn, könnte man - bei weiterer Verfeinerung der Schnittstellentechnologie - theoretisch auch in das Gehirn integrieren (Berger et al. 2005; Berger et al. 2009; Cheung 2007; Stix 2008). Aber das Erscheinungsdatum einer solchen Technologie scheint tatsächlich noch in weiter Ferne.

\section{Datenbanken zur Steuerung von Bewegungen}

Anders sieht es bei den sogenannten BrainComputer-Interfaces aus: Raumzeitlich aufgelöste bioelektrische Signale werden mit aktiven, vollständig implantierbaren und telemetrisch abfragbaren Mikroelektroden zur Steuerung von Computern und Aktuatoren nutzbar (Acharya et al. 2008; Donoghue et al. 2007; Kim et al. 2009). Auch der umgekehrte Prozess - die Verwendung von Datenbanken mit raumzeitlichen sensomotorischen Mustern zur präzisen Steuerung von Bewegungen - erscheint denkbar (Berger et al. 2008). Immerhin sind die zugrunde liegenden mathematischen Modelle offenbar exakt, sonst hätte Sony nicht schon 2007 einen Roboter mit menschenähnlichen Bewegungen konstruieren können (BarCohen, Hanson 2009).

Dennoch: An welchem Punkt ist die Entwicklung auf dem Gebiet der Verstärkung menschlicher (oder übermenschlicher?) Leistungsfähigkeit mit neuroelektronischen Implantaten angekommen? Ist eine ethische Debatte zum „Enhancement“ auf diesem Gebiet überhaupt erforderlich?

Zunächst einmal sollte begriffliche Klarheit herrschen. Der englische Begriff „Enhance- 
ment" [engl.: Erhöhung, Steigerung, Ausdehnung, Erweiterung, Anreicherung, Verbesserung, Vergrößerung] wurde konzeptuell oft als das Gegenstück von „Treatment“ [engl.: Behandlung im medizinischen Sinn] abgegrenzt (Hansson 2005; Merkel et al. 2007). Die intensiver werdende Debatte hat $\mathrm{zu}$ einer tieferen Beschäftigung mit dem Begriff geführt. Insbesondere wurde zu Recht kritisiert, dass z. B. das klassische Doping und die plastische Chirurgie schwerlich unter der Kategorie „Enhancement“" zu klassifizieren sind. Definiert man also „Enhancement" mit Armin Grunwald als eine Verstärkung menschlicher Leistungsfähigkeiten über das für gesunde, begabte Menschen unter optimalen Bedingungen erreichbare Maß hinaus, dann bekommt der Begriff auch im Deutschen ein gewisses Alleinstellungsmerkmal - wenn auch, wie bei jeder anderen Enhancement-Definition, bestimmte Grauzonen verbleiben. Ein Beispiel dafür ist die Schwierigkeit einer exakten Differenzierung zwischen den angrenzenden Kategorien „Doping“ und „Umbau“ (,,alteration"; Grunwald 2009, S. 2).

Im Rahmen dieses Aufsatzes scheint damit eine ausreichend sinnvolle Wortbedeutung gegeben, so dass im Weiteren auf die Apostrophierung des Begriffs verzichten werden darf.

Die Beschäftigung mit dem Begriff allein - sei es in der Öffentlichkeit oder im eher exklusiven philosophischen Diskurs - macht daraus aber noch keine wirklich relevantes Thema, selbst wenn sich bereits fachspezifische Sparten abzugrenzen beginnen (wie „Neuroethik“,
„Biopolitik“ oder „Implantatethik"). In der Tat scheint es hier eine Diskrepanz zwischen echtem wissenschaftlichem Fortschritt und öffentlicher Wahrnehmung auf dem Gebiet des neuroelektronischen Enhancement zu geben.

Eine Internet-Suchanfrage unter Google für die Kombination der Begriffe „Human“, „Enhancement", „Electrodes" und „Ethics“" ergibt 8.290 Einträge; diese sind zumindest im Überblick größtenteils hochrelevant bezüglich des hier behandelten Themas. Die gleiche Anfrage ergibt unter „Pubmed“, dem elektronischen Online-Verzeichnis der „US National Library of Medicine“ (NLM) und dem „National Institute of Health" (NIH) ganze zwei Einträge - einen in japanischer Sprache und einen in der Zeitschrift „The New Atlantis“(Abb. 1).

\section{Motivationslagen}

Die Ursachen für diese Diskrepanz sind vielfältig. Hollywood spielt hier mit publikumswirksamen Science-Fiction-Drehbüchern eine Rolle, ebenso die Unterhaltungsindustrie, clevere Romanciers und sensationsabhängige Massenmedien. Ganz entscheidend für die starke Reflexion des Themas Enhancement in der Öffentlichkeit dürfte aber auch eine emotionale Komponente sein - eine Art Urangst, die Kontrolle über sich selbst, die eigene personelle Identität oder (im kollektiven Rahmen) die Kontrolle über die Menschheitsentwicklung $\mathrm{zu}$ verlieren. Oder noch schlimmer: diese Kontrolle abzugeben an

Abb. 1: Internet-Suchergebnis für die Kombination der Begriffe „Human“, „Enhancement“,
„Electrodes“ und „Ethics“، in Google (Oben) und in Pubmed, dem Literaturrecherche
system des National Institute of Health und der U.S. National Library of Health (unten)

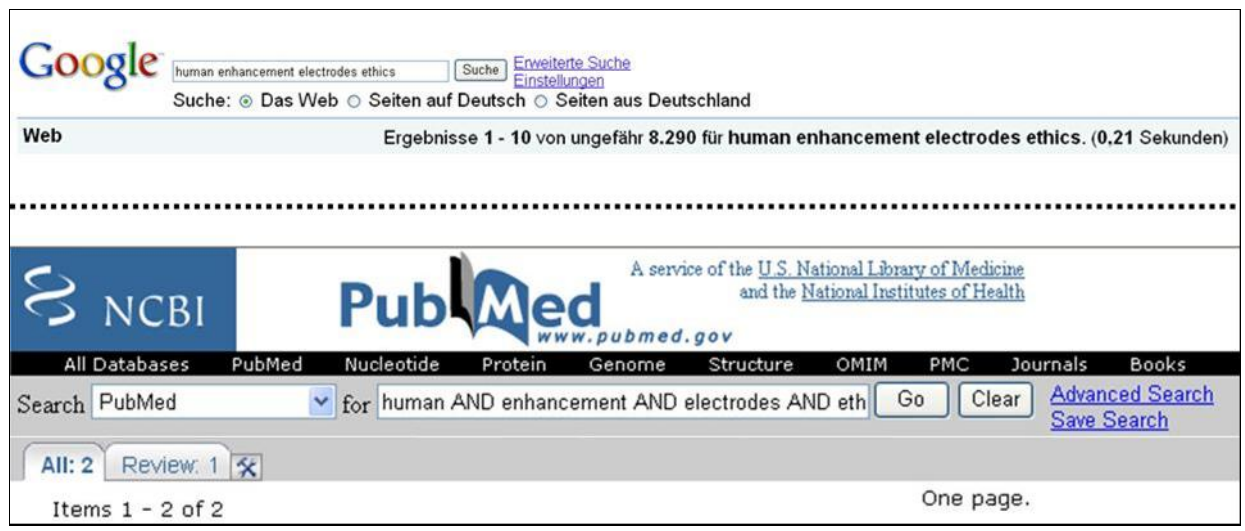

Quelle: Eigene Darstellung/Screenshot Mai 2009 
eine neue Gattung - an den „homo sapiens technificiens". Ein anderer Teil der Bevölkerung (vielleicht ein Drittel?) wiederum erwartet sehnsüchtig gerade diese Verstärkung der eigenen Fähigkeiten. Mancher wünscht sich vielleicht einen siebten Sinn, mit dem er innerhalb Millisekunden zwischen wichtigen und unwichtigen E-Mails unterscheiden kann, ein anderer möchte mit außergewöhnlichen Fähigkeiten für seine Familie den Sprung aus der Armut schaffen, ein Dritter will sich mit einem „Motivationschip“ aus der eigenen Schlaffheit lösen, der Vierte träumt vielleicht tatsächlich von der Weltherrschaft und ein Fünfter möchte Dummheit, Gleichgültigkeit und Kriege abschaffen, indem jeder Einzelne nicht nur mit dem Wissen und der Weisheit der kollektiven Menschheit, sondern auch mit der Verarbeitungskapazität aller Computer der Welt ausgestattet wird. Transhumanisten wie Ray Kurzweil (1999) oder James Hughes (2004) sind womöglich nur die Vorhut dieses öffentlich vorerst eher leise gehaltenen Teils der Erdbevölkerung.

Zurück zu unseren beiden Zwillingsbrüdern. Könnten sich Alex und seine Freunde tatsächlich einem Experiment unterzogen haben, welches ihre kognitiven Fähigkeiten, basierend auf Gedächtnisleistungen, massiv verbessert hat? So massiv, dass sie den Bewertungsmaßstab der Gymnasiumsprüfungen um zwei Notenpunkte nach oben verschieben konnten?

Zur Beantwortung dieser Frage ist die aktuelle Literatur in Bezug auf eine mögliche Verstärkung von Gedächtnisleistungen durch elektrische Stimulation bzw. durch lokal wirksame und applizierbare Substanzen hilfreich. Es ist bekannt, dass Ammonshorn (Hippocampus), Stirnlappen (präfrontaler Cortex) und Verbindungen zwischen diesen Hirnarealen (z. B. Fornix) sowohl an der Bildung von Gedächtnisspuren (Engrammen) als auch am Abruf von Gedächtnisinhalten beteiligt sind. Untersuchungen zur Induktion von Gedächtnisleistungen werden vor allem im Rahmen der Demenzforschung durchgeführt (Stickgold 2006). In der Tat zeigen sich dabei einige ganz erstaunliche Ergebnisse, deren Diskussion an anderer Stelle geführt werden muss, weil sie für die hier angesprochenen ethischen Fragestellungen nur von mittelbarer Bedeutung sind. Fasst man die Befunde dieser neueren Untersuchungen zusammen, dann kommt man bei aller spekulativen Zurückhal- tung $\mathrm{zu}$ der Einschätzung, dass eine gezielte elektrische Stimulation, verbunden mit der Applikation kleinster Mengen bestimmter Substanzen im richtigen Zeitintervall (bezogen auf die Formierung oder den Abruf von Gedächtnisleistungen) wirksame Leistungssteigerungen hervorrufen könnte (Abrari et al. 2009; Ayala et al. 2009; Canals et al. 2008; Hamani et al. 2008; Madronal et al. 2007; Mair et al. 2008; Miasnikov et al. 2008; Ruiz-Medina et al. 2008; Shinoe et al. 2005; Smith et al. 2009; van der Staay et al. 2008). Eine elektrische Stimulation phylogenetisch so alter Strukturen wie des Gleichgewichtssystems hat einen Einfluss auf das räumliche Gedächtnis (Smith et al. 2009). Die Verhaltensänderungen, die durch Stimulation im hypothalamischen Bereich bei Parkinsonpatienten auftreten können, sind inzwischen bekannt. Wendet man diese Methode in anderen Hirnregionen an, dann kann man psychische Erkrankungen wie Zwangsneurosen damit behandeln. Eine elektrische Stimulation im Thalamus kann bei Versuchstieren zielgerichtetes Verhalten, Gedächtnisleistungen und Aufmerksamkeit verstärken (Shirvalkar et al. 2006).

Natürlich bleibt dabei völlig unklar, ob diese Ergebnisse in Zukunft zu einer Implantation von Mikroelektroden oder mikrofluidischen Systemen bei gesunden, voll leistungsfähigen Menschen führen wird. Mit gesundem Menschenverstand würde man davon ausgehen, dass solche Implantate nur dann Interessenten anlocken würden, wenn sie weit wirksamer als nicht-invasive Methoden zur Steigerung der menschlichen Leistungsfähigkeit sind. Handys, Computer, Nachtsichtgeräte, Ultraschallsensoren etc. sind schließlich technologische Hilfen, deren Vermögen unumstritten ist, menschliche Leistungen zu potenzieren.

\section{Verstehen wir die öffentlichen Befürchtungen?}

Halten wir aber für den Moment und unter dem Eindruck der neueren neurowissenschaftlichen Erkenntnisse unseren Geist offen für das vorangestellte Gedankenexperiment und wenden uns der zweiten Hauptfrage zu: Sind unsere „Vordenker", sind Geistes- und Naturwissenschaftler, sind wir selbst wirklich gerüstet für die Diskussion möglicher gesellschaftlicher Veränderungen, die mit der Einführung hochwirksamer 
leistungssteigernder Mikroimplantate im menschlichen Gehirn einhergehen können? Verstehen wir die öffentlichen Ängste und Hoffnungen? Formulieren wir klar, welche neuen Herausforderungen auf uns zu kommen könnten und wie sie zu meistern sind? Oder weigern wir uns, die möglichen Veränderungen überhaupt zu diskutieren, solange konvergierende Technologien uns noch keine überzeugende neurotechnische Schnittstelle zum kognitiven Enhancement auf den Tisch legen? Warten wir einfach nur ab, bis „Memo-Chip Vs. 1.0“ marktreif ist und uns jemand eine implantierbare Bibliothek präsentiert wie Steve Jobbs das neueste iPhone? Überlassen wir einstweilen die Öffentlichkeit (je nach mediengesteuerter Gefühlslage) düsteren Ahnungen oder vorschnellen, unzeitgemäßen Hoffnungen? Sind uns die Transhumanisten soweit enteilt, dass wir uns glücklich schätzen, nicht mehr mit ihnen diskutieren zu müssen, weil wir sie nur für eine neue Art von Sekte halten? Wenden wir uns wieder der fiktiven Geschichte vom Anfang zu.

\section{Erfolg stellt sich ein}

Mit dem exzellenten Prüfungsergebnis in der Tasche bewerben sich Alex und seine Freunde an den besten Hochschulen der Welt und werden mit Kusshand genommen. Weil gravierende, sichtbare Nebenwirkungen - meist in Form intrazerebraler Blutungen - nur bei einem Prozent der Nutzer auftreten, die Implantate aber Gedächtnisbildung und -abruf um den Faktor 10 über das bisher für möglich gehaltene Maximum hinaus steigern, verbreiten sich die Chips quasi über Nacht. Die Herstellerfirma dieser ,"Memo-Chips " wächst rasant, kauft Microsoft und entwickelt sich innerhalb von zehn Jahren zum weltweit größten Unternehmen. Als der Patentschutz entfällt, ist die Monopolstellung schon fixiert. Gleichzeitig beginnt sich die Welt zu verändern. Ein gigantischer technologiegetriebener Wirtschaftsboom stellt alles in den Schatten, was die Menschheit bisher als technologische Revolutionen kannte.

Aufgrund der Leistungsfähigkeit der Chips gibt es für jemanden, der ihn nicht trägt, kaum eine Chance auf eine echte Karriere in Politik, Wirtschaft und Gesellschaft. Zunächst hatte man noch Langzeitstudien gefordert und Implantat- träger von öffentlichen Ämtern für zehn Jahre ausgeschlossen, bevor diese schlüssig bewiesen, dass zum einen diese Art von Diskrimination verfassungsrechtlich nicht vertretbar, zum anderen es auch nicht sinnvoll erscheint, über eine Quotenregelung Schlüsselpositionen nicht an die jeweils Besten zu vergeben. Außerdem werden Datenschutz und individuelle Rechte so verstärkt, dass Implantate nicht mehr registriert und auch bei Bewerbungen oder im Wahlkampf nicht angegeben werden müssen. Arbeitgebern ist es natürlich auf keinen Fall gestattet, ihre Entscheidung, eine Person einzustellen, vom Vorhandensein oder Nichtvorhandensein eines Memo-Chip abhängig zu machen. Schließlich entbrennt noch einmal eine heiße öffentliche Debatte über das Enhancement von Kindern und um die Frage, ab welchem Alter ein Chip implantiert werden darf.

Zunächst gilt ein generelles Verbot bis zur Volljährigkeit, aber durch den Druck besorgter Eltern, die einen Wettbewerbsnachteil ihrer Kinder durch illegale Implantationen bei Mitschülern fürchten, einigt man sich nach mehreren Revisionen des Gesetzestextes, auf ein Enhancement auf Antrag ab dem 14. Lebensjahr, wenn beide Erziehungsberechtigte zustimmen. Der Leistungsdruck in den Schulen wird jedoch so stark, dass es illegale Implantationen vor dieser Altersgrenze gibt.

Memo-Chips der neuesten Generation sind teuer und es scheint zumindest anfangs klar, dass die Kosten von keiner Art Solidarsystem getragen werden sollten. In der Tat gelingt es in den entwickelten Industrienationen zumindest einer relativ breiten Ober- und Mittelschicht, die Kosten selbst aufzubringen - oft allerdings unter Verzicht auf einen Teil des gewohnten Lebensstandards. Die „Dritte Welt" bleibt zu einem großen Teil von der neuen Entwicklung ausgeschlossen - ebenso wie Unterschicht und unterer Mittelstand der ersten Welt. Ein internationales Abkommen vereinbart so etwas wie einen "Artenschutz" für diese Spezies - basierend auf humanistischen Prinzipien und ökonomischen Überlegungen...

Natürlich wird man argumentieren, dass dies alles reine Spekulation ist. Dies gilt aber nur so lange, wie die Grundvoraussetzung fehlt und es keine der in Frage stehenden Implantate gibt. Insofern bleiben unsere gesellschaftlichen 
Normative unangetastet und es genügt auch im Bereich der Forschung und Entwicklung bezüglich konvergierender Technologien, die Grundsätze des Nichtschadens, eines sinnvollen Nutzen-Risiko-Verhältnisses, der individuellen Autonomie und der Gerechtigkeit zu reflektieren, die seit langem - zumindest seit Belmont (The National Commission for the Protection of Human Subjects of Biomedical and Behavioral Research 1979) - unser wissenschaftliches Arbeiten und medizinisches Handelns begleiten und die Jens Clausen in diesem Heft schon klar formuliert hat.

Alle von dieser Grundvoraussetzung abgeleiteten gesellschaftlichen Prozesse erscheinen uns dann aber durchaus plausibel. Und das ist auch der Grund dafür, dass sich Szenarien wie diese im öffentlichen Bewusstsein leicht verankern. Weil so vieles in unserer Welt schwer vorherzusagen ist, tendieren wir heute dazu, technologische Entwicklungen und deren Folgen erst dann normativ einzuordnen und gesamtgesellschaftlich zu diskutieren, wenn sie vollständig erkennbar sind. Dieses Schritt-fürSchritt-Verfahren funktioniert auch gut, solange es sich um Entwicklungen handelt, deren Auswirkungen quantitativ sind und erst allmählich spürbar werden, obwohl auch hier zuweilen die gesellschaftliche Reaktionszeit sehr verzögert sein kann (siehe z. B. Kostenentwicklung im Gesundheitswesen).

Für qualitative, sprunghafte technologische Veränderungen, die unser Menschenbild insgesamt betreffen, dürfte die Schritt-für-SchrittMethode inadäquate Ergebnisse liefern - zumindest für einen gewissen Zeitraum. Ein effizientes, neuroelektronisches Implantat zur Steigerung kognitiver Leistungsfähigkeit würde einen sozialen Druck in Richtung Enhancement erzeugen, welcher - durch den technologischen Imperativ verstärkt - sehr rasche Veränderungen in der Gesellschaft hervorruft. Daher scheint es sinnvoll, die Debatte von Seiten der Wissenschaft nicht weiter zu verschieben, sondern sich in einem vernünftigen Rahmen über Begrifflichkeiten und mögliche Regulierungen zu unterhalten, die losgelöst von einigen HollywoodHorrorszenarien oder Superhelden-Comics eine Basis für einen positiven Umgang mit den Ergebnissen der Neurowissenschaften und ihrer „Verbündeten“ in Mikro- und Nanotechnologie schafft. Nur dadurch wird es sich auch vermei- den lassen, dass die gesamte Forschung auf diesem Gebiet von einem großen Teil der Gesellschaft als ,zwielichtig“ verstanden wird und - zum Nachteil medizinisch-therapeutischer Ansätze - teilweise auf offene Ablehnung stößt.

Mut zur Diskussion, zum Überwinden einer gewissen Trägheit, ein wenig Zeit investiert in Nachdenken und Diskutieren - das alles kann in diesem Zusammenhang eigentlich nur hilfreich sein. Selbst wenn sich eine solche Entwicklung erst in tausend Jahren ergibt oder Enhancement auf eine ganz andere Art und Weise eine Rolle spielt oder andere Probleme in naher Zukunft die volle Aufmerksamkeit der Menschheit erfordern - was hätten wir verloren?

Ängste $\mathrm{zu}$ entfachen ist einfach, sie zu nehmen schwer. Es liegt in unsere Verantwortung als Wissenschaftler und Mediziner, gesellschaftliche Ängste zu adressieren, die sich durch Entwicklungen auf dem Gebiet der Neuroelektronik ergeben. Zuspitzungen, wie die Frage von Hughes, ob es wichtiger sei, dass wir „Menschen“ oder dass wir „Personen“ bleiben, sind wenig hilfreich und führen nur zu Polarisierungen (Hughes 2004).

Vernünftiger erscheint es, in dieser Diskussion Intentionen zu hinterfragen: Was beabsichtigen wir eigentlich, wenn wir menschliche Leistungsfähigkeit steigern wollen? Auf diese Frage gibt es wahrscheinlich so viele Antworten, wie es Lebenseinstellungen gibt. Und nicht alle dürften auf der „dunklen Seite“ zu suchen sein.

\section{Anmerkung}

1) Neben den Cochlea-Implantaten sind auch die Auditorischen Hirnstammimplantate, die immer dann eingesetzt werden können, wenn die Verbindung zwischen Innenohr und Hirnstamm vollständig zerstört ist, inzwischen klinisch etabliert. Als weitere sensorische Implantate sind die Schnittstellen zum visuellen System im klinischexperimentellen Stadium: epi- und subretinale Prothesen, aber auch direkte Verbindungen von Elektroden mit der Sehrinde des Gehirns (kortikale visuelle Implantate). 


\section{Literatur}

Abrari, K. et al., 2009: Post-training administration of corticosterone enhances consolidation of contextual fear memory and hippocampal long-term potentiation in rats. In: Neurobiology of Learning and Memory 91/3 (2009), S. 260-265

Acharya, S. et al., 2008: Decoding Individuated Finger Movements Using Volume-Constrained Neuronal Ensembles in the M1 Hand Area. In: IEEE Neural Systems and Rehabilitation Engineering 16/1 (2008), S. 15-23

Ayala, J.E. et al., 2009: mGluR5 Positive Allosteric Modulators Facilitate both Hippocampal LTP and LTD and Enhance Spatial Learning. In: Neuropsychopharmacology, 34/9 (2009), S. 2057-2071

Bar-Cohen, Y.; Hanson, D., 2009: The Coming Robot Revolution - Expectations and Fears About Emerging Intelligent, Humanlike Machines. New York

Berger, T.W. et al., 2008: Brain-Computer Interfaces: An International Assessment of Research and Development Trends. New York

Berger, T.W.; Glanzman, D.L.; Berger, T.W.; Glanzman, D.L. (Eds.) 2005 Toward Replacement Parts for the Brain: Implantable Biomimetic Electronics as Neural Prostheses. Boston

Canals, S. et al., 2008: Electric stimulation fMRI of the perforant pathway to the rat hippocampus. In: Magnetic Resonance Imaging 26/7 (2008), S. 978-986

Cheung, K.C., 2007: Implantable microscale neural interfaces. In: Biomedical Microdevices. 9/6 (2007), S. 923-938; http://www.springerlink.com/content/yl3 5255557124841/fulltext.pdf (download 24.8.09)

Clausen, J., 2009: Man, machine and in between. In: Nature 457/7233 (2009), S. 1080-1081

Donoghue, J.P. et al., 2007: Neuromotor prosthesis development. In: Med Health RI 90/1 (2007), S. $12-15$

Elder, J.B. et al., 2008: The future of cerebral surgery: a kaleidoscope of opportunities. In: Neurosurgery 62/6 Suppl 3 (2008), S. 1555-1579

Grunwald, A., 2009: Human enhancement - what does "enhancement" mean here? In: Akademiebrief, Europäische Akademie zur Erforschung von Folgen wissenschaftlich-technischer Entwicklungen BadNeuenahr-Ahrweiler GmbH, Nr. 88 (2009), S. 1-3

Hamani, C. et al., 2008: Memory enhancement induced by hypothalamic/fornix deep brain stimulation. In: Annals of Neurology 63/1 (2008), S. 119-123

Hansson, S.O., 2005: Implant ethics. In: Journal of Medical Ethics 31/9 (2005), S. 519-525
Hughes, J., 2004 Citizen Cyborg: Why Democratic Societies Must Respond to the Redesigned Human of the Future. Cambridge, MA

Katz, B.F. 2008 Neuroengineering the Future: Virtual Minds and the Creation of Immortality. Hingham, MA

Kurzweil, R., 1999: Homo s@piens. Leben im 21. Jahrhundert - Was bleibt vom Menschen? Köln

Kim, S. et al., 2009: Integrated wireless neural interface based on the Utah electrode array. In: Biomedical Microdevices 11/2 (2009), S. 453-466; http://www.springerlink.com/content/9g538368426 87284/fulltext.pdf (download 24.8.09)

Madronal, N.; Delgado-Garcia, J.M.; Gruart, A., 2007: Differential Effects of Long-Term Potentiation Evoked at the CA3-CA1 Synapse before, during, and after the Acquisition of Classical Eyeblink Conditioning in Behaving Mice. In: The Journal of Neuroscience 27/45 (2007), S. 12139-12146; http://www.jneurosci.org/cgi/reprint/27/45/12139 (download 24.8.09)

Mair, R.G.; Hembrook, J.R., 2008: Memory Enhancement with Event-Related Stimulation of the Rostral Intralaminar Thalamic Nuclei. In: The Journal of Neuroscience 28/52 (2008), S. 14293-14300; http://www.jneurosci.org/cgi/reprint/28/52/14293 (download 24.8.09)

Merkel, R. et al. 2007. Intervening in the Brain: Changing Psyche and Society. Berlin

Miasnikov, A.A.; Chen, J.C.; Weinberger, N.M., 2008: Specific auditory memory induced by nucleus basalis stimulation depends on intrinsic acetylcholine. In: Neurobiology of Learning and Memory 90/2 (2008), S. 443-454

Rosahl, S.K., 2007: Neuroprosthetics and neuroenhancement: can we draw a line? Virtual Mentor American Medical Association Journal of Ethics 9/2 (2007), S. 132-139

Rosahl, S.K., 2008: Ethical and Societal Implications of Central Nervous System Neuroprostheses. Biomed Tech (Berl) 53/1 (2008), S. 343-346

Ruiz-Medina, J. et al., 2008: Intracranial selfstimulation improves memory consolidation in rats with little training. In: Neurobiology of Learning and Memory 89/4 (2008), S. 574-581

Shinoe, T. et al., 2005: Modulation of synaptic plasticity by physiological activation of M1 muscarinic acetylcholine receptors in the mouse hippocampus. In: The Journal of Neuroscience 25/48 (2005), S.11194-11200

Shirvalkar, $P$. et al., 2006: Cognitive enhancement with central thalamic electrical stimulation. In: Proceedings of the National Academy of Sciences 
of the United States of America 103/45 (2006), S. $17007-17012$

Sinclair, J. et al., 2006: A biohybrid dynamic random access memory. In: Journal of the American Chemical Society 128/15 (2006), S. 5109-5113

Smith, P.F.; Darlington, C.L.; Zheng, Y., 2009: Move it or lose it-Is stimulation of the vestibular system necessary for normal spatial memory? In: Hippocampus [in print]

Stickgold, R., 2006: Neuroscience: a memory boost while you sleep. In: Nature 444/7119 (2006), S. 559-560

Stix, G., 2008: Jacking into the brain. In: Scientific American 299/5 (2008), S. 56-61

Suner, S. et al., 2005: Reliability of signals from a chronically implanted, silicon-based electrode array in non-human primate primary motor cortex. IEEE Transactions on neural systems and rehabilitation engineering 13/4 (2005), S. 524-541

The National Commission for the Protection of Human Subjects of Biomedical and Behavioral Research, 1979: The Belmont Report - Ethical Principles and Guidelines for the Protection of Human Subjects of Research. Office of the Secretary of the U.S. Department of Health, education, and welfare

van der Staay, F.J. et al., 2008: The novel selective PDE9 inhibitor BAY 73-6691 improves learning and memory in rodents. In: Neuropharmacology 55/5 (2008), S. 908-918

White, R.J., 1999: Brain chips: postpone the debate. In: The Hastings Center report 29/6 (1999), S. 4

\section{Kontakt}

Prof. Dr. med. Steffen Rosahl

HELIOS Klinikum Erfurt

Klinik für Neurochirurgie

Nordhäuser Str. 74, 99089 Erfurt

E-Mail: steffen.rosahl@helios-kliniken.de

\section{Ethische Aspekte konvergierender Technologien Das Beispiel Gehirn-Computer- Schnittstellen}

\author{
von Jens Clausen, Universität Tübingen
}

Im Forschungsfeld Gehirn-Computer-Schnittstellen konvergieren so unterschiedliche Technologien wie Mikroelektronik, Nano-, Informations- und Biotechnologie. Die ethischen Fragen bei der Erforschung und Anwendung von konvergierenden Technologien stellen sich bei den Gehirn-ComputerSchnittstellen auf mindestens vier unterschiedlichen Ebenen: der Ebene des Individuums, der Ebene der Gesellschaft, der Ebene des Menschenbildes und der Ebene der Forschungsethik.

\section{Einleitung}

Gehirn-Computer-Schnittstellen (Brain-Computer Interfaces, BCIs) verbinden das (menschliche) Gehirn mit einem Computer. Die Schnittstellen leiten entweder neuro-elektrische Signale aus dem Gehirn ab, oder sie stimulieren spezifische Hirnareale mittels künstlich generierter elektrischer Impulse. Solche Verbindungen zum Signalaustausch zwischen dem Zentralorgan des Menschen und technischen Geräten wecken große wissenschaftliche und therapeutische Hoffnungen. Das zugehörige hochinnovative Forschungsfeld zielt zum einen auf ein besseres Verständnis des Gehirns, seiner Funktionen und der zugrunde liegenden physiologischen Prozesse. Die so gewonnenen Erkenntnisse münden zum anderen dann gegebenenfalls in die Entwicklung neuer medizinischer Verfahren und Geräte für Diagnostik und/oder Therapie.

Der Einsatz von Gehirn-ComputerSchnittstellen ist mit einer ganzen Reihe von ethischen Fragen verbunden, die von Beginn an - so meine These - in Forschung und Entwicklung dieser Geräte berücksichtigt werden müssen. Da in diesen Geräten so unterschiedliche Forschungsgebiete wie die Mikrosystemtechnik, die Informations-, Bio- und Nanotechnologie sowie Neurophysiologie und Anatomie konvergieren, sind Gehirn-Computer-Schnittstellen besonders geeignet, an ihnen die ethischen Aspek- 